\title{
Advanced Modulation Formats and MLSE Based Digital Signal Processing for 100Gbit/sec Communication Through Optical Fibers
}

\author{
Albert Gorshtein and Dan Sadot \\ Ben Gurion University of the Negev \\ Israel
}

\section{Introduction}

Modern telecommunications infrastructure is based on optical fibers for data transmission, due to their higher bandwidth and low attenuation. Today, optical fiber is starting to reach apartment buildings and even private homes, and delivers wideband services such as Triple Play (a Triple Play service bundles high-speed Internet access, television, and telephone service over a single broadband connection).

There is a real growing need for data rates of $100 \mathrm{Gbit} / \mathrm{sec}$ and beyond in the access, enterprise, metro, regional and long haul markets. Therefore, next generation transmission systems must be employed, otherwise the optical infrastructure will become overloaded and exceed its current capacity.

Increasing the bit rate, in turn, makes a transmitted data signal be more vulnerable to transmission impairments inherent to an optical fiber, e.g., chromatic dispersion (CD), polarization mode dispersion (PMD), fiber non-linearity (FNL), concatenated optical filtering etc., and noises produced by optical amplifiers and receiver front end electronics. In particular, increasing the bit rate to 100Gbit/sec and above, based on intensity modulation (IM) with direct detection (DD) alone and using a simple hard decision (HD) scheme, leads to dramatic reduction in transmission range and system performance degradation. The main reason for the latter two phenomena is significant shortening of the pulses representing the transmitted bits, which implies broadening of the signal spectrum. It is a well known fact that, the higher the bandwidth of the signal is, the more it suffers from transmission impairments. For example, both CD and PMD cause inter-symbol interference (ISI), making it more difficult to distinguish between the transmitted levels of logical one and logical zero.

Therefore, reliable transmission over sufficient distance at such a high rate (100Gbit/sec and above), can be achieved by both using digital signal post-processing (DSP) and employing advanced modulation formats. Moreover, the quality of the received (and processed) signal may be significantly improved by using (digital) forward error correction (FEC).

Each of these three techniques strives to improve the system performance in its own way. Using advanced modulation formats typically results in a narrower transmitted signal spectrum, effectively increasing the immunity to ISI. This can be achieved, for example, by 
transmitting more than one bit in each symbol. The main drawback of this approach is, the more symbols are in the alphabet (the more bits are in each symbol), the harder for the receiver to distinguish between them. Furthermore, the more complicated chosen modulation format is, the more complex and expensive the electronic and optical component at both transmitter $(\mathrm{Tx})$ and receiver $(\mathrm{Rx})$ side are.

The DSP reduction of ISI can be divided into two main categories. The first one, termed equalization, uses linear (feed forward - FFE) filters and already made decisions (decision feedback - DFE), in order to undo the combination of all the distortions that caused ISI, according to a predetermined criterion, such as zero forcing (ZF), minimum mean square error (MMSE) etc. Typically such equalizers operate in an adaptive manner, updating the filter(s) coefficients from time to time, such that the adaptation rate is greater than the temporal changes in the channel. As an example, the CD phenomenon is constant for a given link, whereas the PMD effect is stochastic in nature, when the amount of PMD may vary over time constants of $1 \mu \mathrm{sec}$ to $1 \mathrm{msec}$. The second category is called maximum likelihood sequence estimation (MLSE), which uses a different approach. MLSE exploits the statistics of the received signal, whereas the decisions are made on the whole transmitted sequence rather than on every single bit. While the complexity of the MLSE is significantly greater than of linear and nonlinear equalizers from the first category, it is proven to be the best optimal tool for combating ISI in a communication system (Proakis, 1995). MLSE can also be made adaptive, if the statistics that is used for decoding is updated fast enough to follow the changes in the channel. In this chapter we will focus on DSP, that implements MLSE decoders, rather than FFE and DFE.

Prior to performing the DSP, the received signal has to be transferred into the digital domain. This task is done by means of analog-to-digital conversion (ADC), that turns out to be a major bottleneck of communications systems, transmitting 100Gb/sec and more. The common practice is taking two samples per every symbol (also called oversampling) with successive equalization. Since the task of the decision device in the receiver is to estimate the transmitted symbols, it is enough to sample each symbol only once, but at the optimal sampling instants. Oversampling eliminates the sensitivity to the sampling phase, though requires more complex and power hungry ADCs. To the best of the authors' knowledge, the highest ADC sampling rate commercially available today is $64 \mathrm{~Gb} / \mathrm{sec}$. Even ADCs with the rates of $28-32 \mathrm{~Gb} / \mathrm{sec}$ are still state of the art nowadays. Therefore, it is important to put more effort in the digital domain, and develop such post processing algorithms that enable reduction of sampling rate. Moreover, sampling the received signal at the symbol rate without preceding filtering violates the Nyquist sampling criterion, causing aliasing effect that results in performance degradation. On the other hand, using anti aliasing filter (AAF) prior to symbol rate sampling introduces heavy low pass filtering (LPF) which, in turn, causes heavy inter symbol interference. In this chapter we propose symbol rate sampling with the use of AAF, followed by equalization of transmission impairments and MLSE to compensate for ISI.

The immunity of any communication system can be increased at the expense of adding a few percents (typically $7 \%$ to $30 \%$ ) of overhead symbols that are not part of the information that is needed to be transmitted. This effectively increases the transmitted bit rate and hence the bandwidth of the transmitted signal. This overhead, called code bits, is used by the FEC system to detect and correct errors. Common FEC schemes available today are capable of 
decreasing the bit error rate (BER) from $10^{-2}$ or $10^{-3}$ to $10^{-9}$ or even $10^{-12}$, depending on many factors such as amount of overhead, the coding rules (or coding gain), number of iterations and so on. However, FEC needs a certain BER at its input, in order to be capable of carrying out the correction, called pre-FEC BER. Throughout this chapter, it is assumed that the transmission system incorporates FEC, thus most of the results will be presented in a form of optical signal to noise ratio (OSNR) or optical input power that is required to achieve a pre-FEC BER value of $10^{-3}$.

Fiber optics communication systems can be categorized by many different factors like transmission distance, bit rate, modulation and detection scheme, post processing and so on. However, the categories presented above, are not entirely independent from each other. As in any other communication system, the appropriate combination of the above characteristics depends on a particular application. We roughly divide the systems by the required transmission distance: "extended short reach" (up to $80 \mathrm{~km}$ ), metro (up $1000 \mathrm{~km}$ ) and long-haul systems (more than $1000 \mathrm{~km}$ ). Depending on this division, we propose the system configuration for $100 \mathrm{~Gb} / \mathrm{sec}$ transmission, including modulation format and successive MLSE based DSP, and analyze the expected system performance.

This chapter is organized as follows. Section 2 will briefly discuss the MLSE processing principles. Sections 3, 4 and 5 will present the aforementioned systems analysis, associated DSP algorithms and expected performance of the proposed modulation formats for short reach, metro and long-haul systems respectively. In section 6 we will draw out the conclusions of the presented work.

\section{Optical fiber as a communication channel}

Propagation of electrical field in the optical fiber can be described by the non-linear Schrodinger equation (Agrawal, 2002):

$$
\frac{\partial E(t, z)}{\partial z}+\frac{j}{2} \beta_{2} \frac{\partial^{2} E(t, z)}{\partial t^{2}}+\frac{\alpha}{2} E(t, z)=j \gamma|E(t, z)|^{2} E(t, z)
$$

where $E(t, z)$ is the incoming electrical field, $\alpha$ is an attenuation coefficient, $\gamma$ is a nonlinearity coefficient and $\beta_{2}$ is a group velocity dispersion (GVD) coefficient, accounting for chromatic dispersion. When the total input optical power to the standard single mode fiber (SSMF) is not too high (less than $5 \mathrm{dBm}$ ) the non-linear term in (1.1) is negligible, and it may be assumed that the fiber is operating in the linear regime. Under this assumption, (1.1) is reduced to a linear Schrodinger equation:

$$
\frac{\partial E(t, z)}{\partial z}+\frac{j}{2} \beta_{2} \frac{\partial^{2} E(t, z)}{\partial t^{2}}+\frac{\alpha}{2} E(t, z)=0
$$

Thus, the channel can be modeled with help of transfer functions accounting for two effects: $\mathrm{CD}$ and loss.

\subsection{Chromatic dispersion}

The essence of chromatic dispersion is in the fact that different frequency components of the transmitted signal travel through the fiber at different velocities. The effect of chromatic 
dispersion is deterministic since it is completely defined by physical parameters of the fiber. The baseband transfer function, describing the CD phenomena for a fiber of length $L$ is obtained by solving (1.2) for $\alpha=0$ (assuming that loss is compensated by proper optical amplification), yielding:

$$
H_{C D}(f)=\exp \left\{-j \beta_{2}(2 \pi f)^{2} \frac{L}{2}\right\}
$$

Any type of modulated data has a nonzero spectral width, due to the presence of information, which occupies a range of frequencies that is roughly of the same order of magnitude as the bit rate itself. These different spectral components of modulated data travel at different speeds down the fiber. In particular, for digital data modulated on an optical carrier, CD leads to pulse broadening, which, for a given bit rate and modulation format, limits the distance that signal can propagate through, and the appropriate levels can still be distinguished. In other words, the effect of chromatic dispersion is cumulative and increases linearly with transmission distance.

Moreover, it can be shown that this maximal transmission distance $L_{D}$ (without compensation) is proportional to the ratio of symbol duration $T_{s}$ and the signal bandwidth $B W$ :

$$
L_{D}=\frac{T_{S}}{k \cdot D \cdot B W}
$$

where the coefficient $k$ depends on the allowed performance penalty and modulation format, and $D$ is a dispersion parameter that related to $\beta_{2}$ as follows:

$$
D=-\frac{2 \pi c}{\lambda_{0}^{2}} \beta_{2}
$$

$\lambda_{0}$ being the wavelength of the optical carrier. Given the bit rate and modulation format, the amount of chromatic dispersion depends entirely on the physical characteristics of the channel: the dispersion parameter $D$ and the fiber (or uncompensated span) length $L$. Therefore, based on (1.4) it convenient to combine these two together, and measure the amount of chromatic dispersion in the link by the $C D$ parameter, defined as:

$$
C D \triangleq D \cdot L=\frac{T_{s}}{k \cdot B W}
$$

In the sequel, the performance of the examined systems will be presented as a function of $C D$.

The compensation of chromatic dispersion can be done either in the optical or in the electrical domain. Sometimes, the combination of both techniques is used. In the optical domain the compensation is typically performed with help of dispersion compensation fibers (DCF) and/or dispersion compensation modules (DCM), both having a dispersion parameter with the opposite sign to the corresponding SSMF span to be compensated. DCF and DCM provide constant CD compensation. For $10 \mathrm{~Gb} / \mathrm{sec}$ systems they are typically placed every $80 \mathrm{~km}$ in the channel, and introduce additional loss, which has also to be 
compensated by optical amplification. The last span length, however, may vary, depending on specific geography and topology of the field. Hence, for this last lag, two options exist. First one is to put a more complex (and expensive) tunable dispersion compensator (TDC). And a second one is to design the system such that no compensation is required or alternatively, the compensation is carried out in the electronic domain.

\subsection{Polarization mode dispersion}

As a result of the fiber's birefringence different modes of polarization split and travel in different velocities causing pulse spreading. The effect of light propagation in a birefringent fiber is generally termed polarization mode dispersion (PMD). Birefringence occurs due to changes in fiber geometry (symmetry), which may be caused by either manufacturing imperfections, temperature variations or mechanical vibrations. Therefore, on contrary to $\mathrm{CD}, \mathrm{PMD}$ has a stochastic nature. In case of polarization multiplexed transmission the effect of PMD can be summed up in the following things. In addition to different delays, the transmitted electrical field undergoes polarization rotation, meaning that the electrical field at the fiber output may have a different state of polarization (SOP), (Kaminow \& Li, 2001). Hence, when looking at the received electrical fields in some fixed $\mathrm{SOP}$, there is a linear combination of the incoming fields from both polarization modes. In other words, PMD results in intra-channel crosstalk that cause a pulse spreading. In fact, in this case an SSMF can be viewed as multiple input - multiple output (MIMO) system (with two inputs and two outputs). Therefore, it can be described by the transfer function matrix (in the frequency domain), called the Jones matrix, which in the absence of polarization dependant loss and in case of first order PMD has the form (Ip \& Kahn, 2007):

$$
\mathbf{H}_{\mathbf{P M D}}(f)=\left(\begin{array}{cc}
\cos \psi & \sin \psi \\
-\sin \psi & \cos \psi
\end{array}\right)\left(\begin{array}{cc}
e^{j 2 \pi f \frac{\tau}{2}} & 0 \\
0 & e^{-j 2 \pi f \frac{\tau}{2}}
\end{array}\right)\left(\begin{array}{cc}
\cos \psi & -\sin \psi \\
\sin \psi & \cos \psi
\end{array}\right)
$$

where $\tau$ is the differential group delay (DGD) parameter and $\psi$ is the angle between the SOP of the electrical field at the fiber input and the principal state of polarization (PSP) of the fiber (Kaminow and Li, 2001). Thus, the capacity of transmitted information can be doubled, when sending two independent data streams on different polarization modes of the SSMF.

In systems, where the information is modulated only on a single polarization of the optical fiber, equation (2.1) reduces to (Kaminow and $\mathrm{Li}, 2001$ ):

$$
H_{P M D}(f)=\sqrt{\rho} \exp \{-j \pi f \tau\} \cdot \hat{x}+\sqrt{1-\rho} \exp \{+j \pi f \tau\} \cdot \hat{y}
$$

where $\rho$ represents the power splitting ratio. Moreover, if in addition to single polarization modulation the signal is detected directly with help of photo-detector (PD), the resulting photo-current would take the form:

$$
I_{e l}(t)=R \cdot\left\|\mathrm{E}_{\mathrm{r}}(t)\right\|_{2}^{2}=R \cdot\left|E_{r, x}(t)\right|^{2}+R \cdot\left|E_{r, y}(t)\right|^{2}
$$


where $R$ represents the PD responsivity, $E_{\mathrm{r}}(\mathrm{t})$ is the incident optical field vector having non-zero $\hat{x}$ and $\hat{y}$ components $E_{r, x}(t)$ and $E_{r, y}(t)$ respectively.

\subsection{Optical amplification and amplified spontaneous emission noise}

To compensate for the attenuation described by $\alpha$ in (1.2), especially in transmission for long distances (long-haul), the propagated optical field is generally amplified by means of an erbium-doped fiber amplifiers (EDFAs) every $L_{A}$ kilometers. For example, in a SSMF having $\alpha=0.2\left[\frac{d b}{\mathrm{~km}}\right]$, the EDFAs need to be placed every $L_{A}=80[\mathrm{~km}]$ for $10 \mathrm{Gbps}$ transmission. In addition to the beneficial high gain that remains constant for relatively large wavelength range, EDFA produces and amplifies a spontaneous emission, which is undesirable and can be treated as noise, generally referred as ASE noise. The power spectral density (PSD) of the ASE noise is nearly constant and given by (Agrawal, 2002):

$$
S_{n}(f)=\frac{N_{0}}{2}=\frac{1}{2} n_{s p} \frac{h c}{\lambda_{0}} \frac{(G-1)}{G}
$$

where $n_{s p}$ is the spontaneous emission factor (or population inversion factor), G represents the EDFA gain, $\frac{h c}{\lambda_{0}}$ is the photon energy at the wavelength $\lambda_{0}$, corresponding to the central frequency of the optical carrier $f_{c}, h$ being the Plank constant and $c$ being the speed of light. The ASE noise in optically amplified systems, particularly in those that have more than one EDFA in the link, is dominant over all other types of noises, produced in the receiver (thermal noise, shot noise, dark current noise), due to the repetitive generation and amplification of ASE noise in each span. To reduce the effect of ASE, optical filters, centered at $\lambda_{0}$ with bandwidth $B_{O}$, are used.

\section{MLSE processing principles}

This section presents MLSE processing principles suitable for fiber optical communication systems. Especially, due to the presence of PMD the optical channel is considered to be nonstationary and adaptive equalization is required. When the histograms, which serve as channel estimators, are updated faster than channel variation rate, successful variations tracking can be achieved. Moreover, since channel estimation without resorting to training sequence is beneficial, a novel blind channel estimation technique is proposed.

\subsection{General decoding principle}

Maximum likelihood sequence estimation (MLSE) is considered to be non-linear equalization technique. To explain the main idea that is behind the MLSE processing trellis diagrams are often used. The example of 4-state trellis diagram is presented on Fig. 1.

In this example two bits of the channel memory are assumed, i.e. current sample is affected by two previous and the current bit. The two previous bits define the channel state, and a conjunction of a state with a current bit defines a branch. The arrows in the trellis represent 


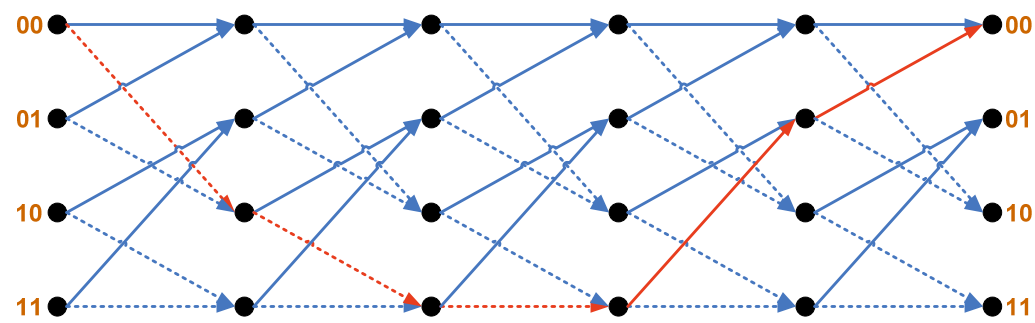

Fig. 1. 4-state trellis diagram example

the transition from one state to another, while a transition corresponding to a ' 0 ' bit is drawn with solid line and a transition corresponding to a ' 1 ' is represented by a dashed line. To each branch in the trellis we assign the number called "branch metric", which is dependent on channel and noise statistics. Branch metric describes in some sense the probability of a corresponding transition. Furthermore, one can define a "path metric" which is the sum of corresponding branch metrics for a certain path, when a bit sequence with a smallest path metric is the most probable to be transmitted.

The key idea of the MLSE processor is to choose the path with the smallest metric, and produce the most likely sequence by tracing the trellis back (Proakis,1995). For sequence of length $N$ there are $2^{N}$ possible paths in the trellis, therefore an exhaustive comparison of the received sequence with all valid paths is inefficient task, becoming non feasible for channels with long memory. However, noting that not all paths have the similar probabilities (or metrics) when proceeding through the trellis, there exists an efficient algorithm, called Viterbi algorithm, that limits the comparison to $2^{K}$ "surviving paths", $K$ being the channel memory length, independent of $N$, making the maximum likelihood principles to be practically feasible. The main idea of the Viterbi algorithm: from two paths entering the trellis node, the path with the smallest metric is the most probable. Such a path is called the "surviving path", and we need to store only surviving paths with their running metrics.

\subsection{Metrics generation and update}

Maximum likelihood sequence detection is the proven to be the most effective technique for mitigating optical channel impairments such as chromatic dispersion and polarization mode dispersion (Foggi et al., 2006). In order to successfully apply this technique, it is mandatory to estimate some key channel parameters needed by the Viterbi processor.

Channel estimation methods can be classified as parametric and non-parametric. Parametric methods assume that the functional form of the probability density function (PDF) is known, and only its parameters need to be estimated. However, non-parametric methods do not assume any knowledge about the PDF functional form or its parameters. There are two most common methods used in practice for channel estimation: method of moments (MoM) and histogram estimation method.

Method of moments is considered to be parametric, therefore it assumes that the functional form of the PDF is known and only its moments need to be estimated. When the dominant noise mechanism in the optical system is thermal, like in optically unamplified links, the 
conditional PDF of the received sample $x_{n}$, given that $\mu_{k}$ is transmitted, is assumed to be Gaussian with $\sigma_{n}^{2}$ being the variance of the noise (Proakis, 1995):

$$
f_{\text {channel }}^{\text {Gaussian }}\left(x_{n} \mid \mu_{k}\right)=\frac{1}{\sqrt{2 \pi \sigma_{n}^{2}}} \exp \left\{-\frac{\left(x_{n}-\mu_{k}\right)^{2}}{2 \sigma_{n}^{2}}\right\}
$$

In this case, only first and second moments need to be estimated. Another case of interest is ASE limited channel. As stated above the noise in such a channel becomes signal dependent and the functional form of the conditional PDF of the received sample $x_{n}$, given that $\mu_{k}$ is transmitted, can be approximated by a non-central Chi-square distribution with $v$ degrees of freedom (Agazzi et al., 2005):

$$
f_{\text {channel }}^{\text {ASE }}\left(x_{n} \mid \mu_{k}\right)=\frac{1}{N_{0}}\left(\frac{x_{n}}{\mu_{k}}\right)^{\left(\frac{\nu-1}{2}\right)} \exp \left\{-\frac{x_{n}+\mu_{k}}{N_{0}}\right\} I_{v-1}\left\{2 \frac{\sqrt{x_{n} \mu_{k}}}{N_{0}}\right\}
$$

where $N_{0}$ is power spectral density of the ASE noise given by (2.4), and $I_{\{.\}}$is the modified Bessel function of the first kind. It is clear that in (3.2) $N_{0}$ and $v$ need to be estimated. More information about parametric estimation is given in (Agazzi et al., 2005; Foggi et al., 2006).

Histogram method does not assume anything about the PDF of the received samples. In this method $M^{N_{i s i}+1}$ histograms are collected, where $M$ represents the vocabulary size of the transmitted symbols and $N_{i s i}$ is the number of the most resent previous symbols that affect the current symbol, i.e. the channel memory length assumed by the algorithm. The received signal is assumed to be quantized to $N_{A D C}$ bits; therefore each histogram consists of $2^{N_{A D C}}$ bins, where $N_{A D C}$ is a design parameter. Notice that each histogram can be uniquely associated with a branch in the trellis diagram of the receiver. Assuming that the number of signal samples collected is large; the histogram (normalized so that the sum of all its bins is unity) is an estimate of $f_{\text {channel }}\left(x_{n} \mid \mu_{k}\right)$. The histogram is updated iteratively, based on the observed samples and the decision bits at the output of the MLSE decoder.

Finally, branch metrics are obtained by taking the natural logarithm of the estimated/assumed PDF. For a transmitted sequence of length $N$ the MLSE decoder chooses between $M^{N}$ possible sequences that minimize the (path) metric:

$$
m_{r}=\sum_{n=1}^{N}-\ln \left\{f_{\text {channel }}\left(x_{n} \mid \mu_{k}\right)\right\}
$$

The estimated bit sequence is determined by tracing the trellis (like in Fig. 1) back, based on the minimal path metric (3.3).

In optical fiber systems, the purpose of the MLSE is to combat ISI stemming from CD and PMD. While CD is a deterministic phenomena for a given link, PMD is stochastic in nature, therefore an adaptive equalizer, that performs PMD tracking is required. Moreover, the adaptation properties of the MLSE can be also exploited for CD compensation when the amount of $\mathrm{CD}$ is not perfectly known. Basically, as will be explained in the next sections expensive tunable optical dispersion compensation may be replaced by the adaptive MLSE. 
This type of operation, without knowing any initial information about the channel is termed blind equalization.

The operation of the proposed blind MLSE equalization scheme can be divided into two main modes: initialization and steady state operating mode. At the initialization mode, there are two main tasks to be fulfilled. First, initial coarse histograms estimation representing the channel is required. This can be achieved by using a set of predetermined histograms representing different channels, as will be explained in the next subsection.

Starting from this initial "guess", an iterative procedure is activated in order to fine tune the channel estimation. At each iteration data decoding is performed by applying the Viterbi algorithm based on a previous estimation of the channel. This iterative histograms estimation is obtained in a decision directed manner; it is assumed that the receiver operates at a sufficiently low BER. The calculation of the channel estimator (histograms) for the next step is then based on the observation-decision pairs available from the current iteration. Consequently, the histograms are obtained from current observation set, while the decoded symbols are used as a 'training sequence' for the attribution process. This process is repeated until a convergence criterion is being met. Even though theoretically the convergence in a decision directed mode is not guaranteed, in practice the proposed method was found to be extremely robust as it converges in a very wide set of channel conditions.

To ensure sufficient tracking, the histograms must be updated fast enough as compared to temporal variations of the channel. Since PMD changes in the scale of $100 \mu \mathrm{sec} \div 1 \mathrm{msec}$, the histogram adaptation rate must be at least ten times faster, meaning that every $10 \mu \mathrm{sec}$ a new set of metrics must be obtained.

\subsection{Initial metrics}

The key function that enables the blind MLSE processing is the proper choice of the initial metrics. In fact, since only coarse channel representation is needed, it may be assumed that the branch histograms have nearly Gaussian shape and differ from each other only by the mean and variance. The mean values depend on the channel memory length and the vocabulary size in the Rx side $M$. To ensure proper operation, the decoder must be designed such that the channel memory length $K$ is at most as the memory length of the decoder $N_{i s i}$. In this case there are $M^{N_{i i}+1}$ branches, whereas the variance of each histogram is associated with the noise power that is present in the corresponding combination describing the branch. In memoryless channel with binary vocabulary there are two histograms, representing the corresponding conditional PDFs, and simple hard decision scheme can be used. When $M=2$ and channel memory of one symbol $(K=1)$ there are four distinct histograms, having four different mean values. Generally, when $K<N_{i s i}$, the actual number of histograms in the given MLSE decoder is constant, $M^{N_{i i}+1}$, and consists of $M^{K+1}$ different groups, while all the members of such a group are identical. Continuing the example, if $N_{i s i}=4$ there are 32 branches, which can be divided into 4 groups, associated with the four different mean values mentioned above. Thus, the set of initial metrics for the given $M$ and $N_{i s i}$ is finite and easy to obtain by distributing $M^{K+1}$ means uniformly, and leaving the fine tuning to the iterative convergence process. Another factor that increases the number of initial metrics in the bank is the variance of the branches. Taking into account that assigning higher variance than actual does not affect the performance, the number of initial metrics can be further reduced by assigning the same (high) variance to all the branches. 
Initial metrics determination is summarized on Fig. 2.

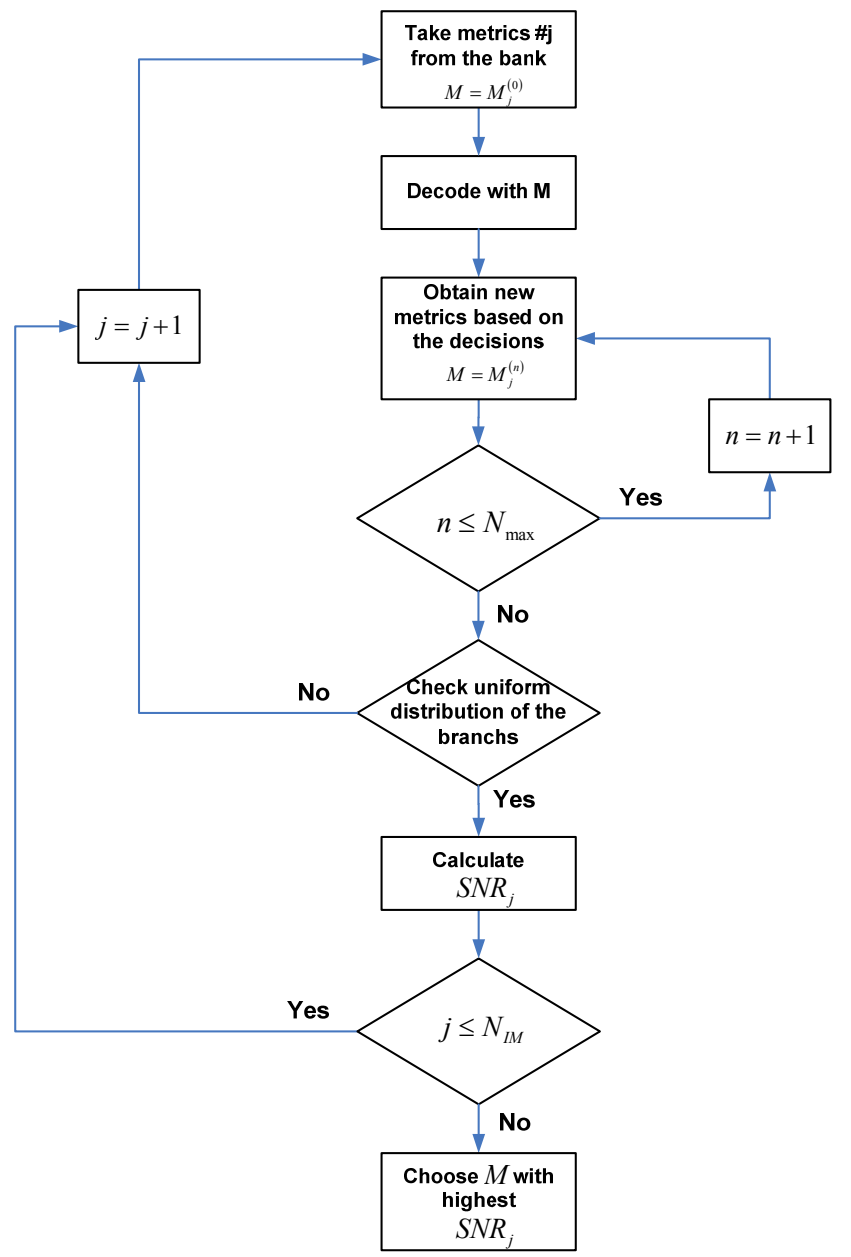

Fig. 2. Initial metrics determination procedure

The initialization phase typically needs to be done "once in a lifetime" during system start up, or after reset, and typically requires only a few iterations, where each iteration uses a couple of thousands of symbols. Once convergence is achieved, the equalizer switches to the steady state operating mode, where the histograms are updated continuously, based on the previously made decisions. In order to verify that the system remains in steady state mode, a uniform distribution of Viterbi trellis branches, represented by the histograms, is required. In addition, a sufficiently low BER value condition must be met during this operating mode. Otherwise, an interrupt signal is generated and sent to the control processing unit (CPU).

To choose the best histogram of all the initial metrics in the bank is the best, the following signal-to-noise ratio (SNR) criterion is used: 


$$
S N R=\frac{\sum_{i=1}^{N_{b r}} \mu_{i}^{2}-\frac{1}{N_{b r}}\left(\sum_{i=1}^{N_{b r}} \mu_{i}\right)^{2}}{\sum_{i=1}^{N_{b r}} \sigma_{i}^{2}}
$$

In (3.4) $\mu_{i}$ and $\sigma_{i}^{2}$ represent the mean and the variance of branch number $i$, which are estimated from the histograms, and $N_{b r}$ is the total number of branches (histograms). Actually, this is the same criterion as in (Gorshtein et al., 2010), but shown to be working with much wider set of channels describing a signal coming either from coherent or direct detection scheme.

\section{4. "Extended short reach" transmission}

\subsection{Introduction}

In most common $10 \mathrm{~Gb} / \mathrm{sec}$ systems the link length is limited by chromatic dispersion and stands on $80 \mathrm{~km}$. When longer reach is desirable, dispersion compensation fibers are used, and the system becomes power limited. This power limitation is been overcome by optical amplifiers, which are typically placed every $80 \mathrm{~km}$, and compensate for both attenuation in the fiber and the DCF together with insertion loss (IL) of the DCF. A short reach transmission system is defined here, as a system that does not require any optical amplification. Thus, due to CD limitation typical length of the link (at 10Gb/sec transmission) is about $80 \mathrm{~km}$.

Generally, there are two main reasons that lead to a degradation of signal quality: ISI and noise. Due to the lack of optical amplifiers in short reach systems, the dominant noise mechanisms are thermal noise and shot noise (Agrawal, 2002). Both noises are independent of the optical channel length and added to the received signal during the optoelectronic conversion. It is worth to note, that noise, is always present in the frequency band occupied by the signal, and cannot be eliminated or separated from the signal by any means, without hearting the signal itself.

However, the other source of received signal quality degradation, ISI, can be theoretically eliminated completely (given no noise), by employing the MLSE technique, described in the previous section. Moreover, the ISI, being generated mainly by the channel itself (CD, PMD), is an increasing function of fiber length (Agrawal, 2002). Since the received signal quality scales with distance, particularly in short range transmission systems it is desirable to reduce the overall system cost as much as possible. Therefore, it is preferable to use inexpensive optics, leaving in the 'battlefield' only the most simple intensity modulation and direct detection (IMDD) systems. In fact, for a given data rate (of $100 \mathrm{~Gb} / \mathrm{sec}$ in our case), tradeoff can always be done between the overall system cost and complexity versus performance and spectral efficiency.

From equation (1.4) it is clear that the less the bandwidth of a digital signal at a given bit rate, the longer the propagation distance in the fiber. Based on this observation, it is proposed to reduce the bandwidth of the transmitted signals at the transmitter side with use of previous generation $10 \mathrm{G}$ optical components. Furthermore, since the receiver bandwidth is lower, less noise will be added to a signal. In addition, 10G avalanche photo diode (APD) can be used for better sensitivity. Note, that to the best of the authors' knowledge APDs for 
higher transmission rates still do not exist (at least up to June, 2011). However, these potential benefits do not come for free, since such heavy low-pass filtering by itself introduces ISI. To compensate for this effect, together with the ISI stemming from CD and PMD, the MLSE decoder is used in each link. Due to such a short reach and relatively wide transmitted pulses the PMD tolerance is of the second order and only CD tolerance is investigated.

To achieve a target of $112 \mathrm{~Gb} / \mathrm{sec}$ (including $12 \%$ FEC overhead), a four-wavelength transmission is proposed, where each lambda (wavelength) carries a data of $28 \mathrm{~Gb} / \mathrm{sec}$. In light of the aforesaid, the following novel modulation formats (with MLSE detection) will be examined: reduced bandwidth on-off-keying (RBW-OOK) and reduced bandwidth duobinary (RBW-DB) modulation formats.

\subsection{System model}

Block diagram of the proposed system is presented in Fig. 3.

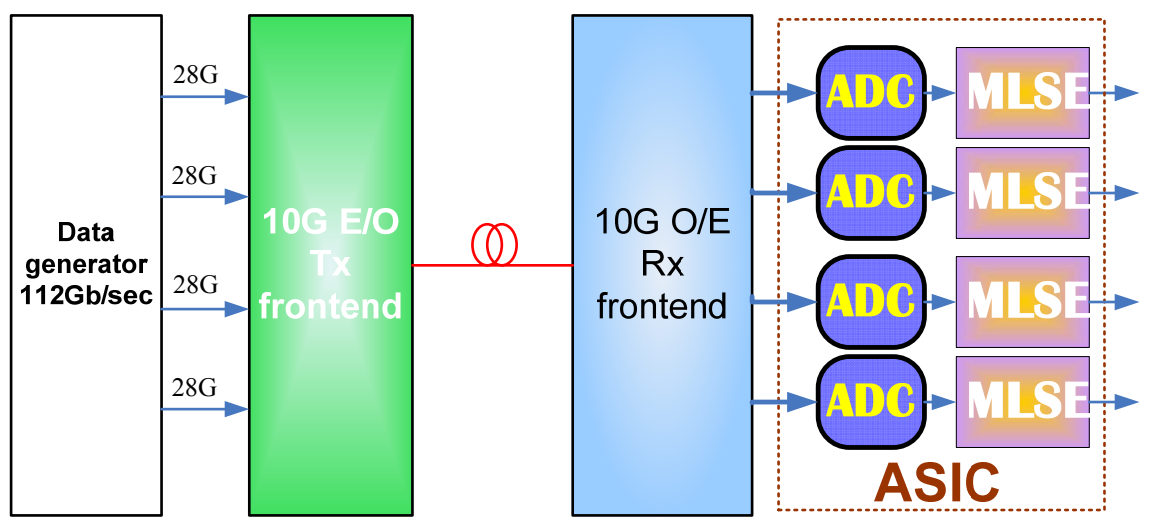

Fig. 3. High- level block diagram of the "extended short reach" transmission system

Four parallel lanes, 28Gb/sec each, are transmitted with help of four different carrier waves, designated by $\lambda_{1}$ to $\lambda_{4}$. The electro-optic (E/O) transmitter (Tx) frontend consists of four identical pairs of $10 \mathrm{G}$ electrical drivers and 10G optical modulators, whereas the modulator type depends on the modulation format.

RBW-OOK can be generated either by modulating the 10G laser directly or by keeping the laser producing a continuous wave $(\mathrm{CW})$ and using either an external (10G) electroabsorption modulator (EAM) or (10G) Mach-Zehnder modulator (MZM). DB modulation format is generated with help of 10G MZM biased at null point. However, there are additional degrees of freedom: the bandwidth and order of the combined filter representing the cascade of modulator and driver filters. The resulting four signals are multiplexed into a short fiber link.

To achieve higher transmission distance with OOK formats chirped MZM or EAM with chirp parameter $c=-0.7$ and $c=-1.2$ are used. In the DB case the chirp has a destructive effect on the encoded signal, hence a zero chirp MZM is used. 
The optical receiver $(\mathrm{Rx})$ front end is the same for both OOK and DB. First, the four subchannels are demultiplexed from the link, and then opto-electronic $(\mathrm{O} / \mathrm{E})$ conversion is carried out by four (identical) photodiodes. The resulting photo-currents are sampled at $28 \mathrm{Gsamples} / \mathrm{sec}$ rate and quantized with four 5-bit resolution analog-to-digital converters (ADCs). Then MLSE detection, with channel memory of 4, is performed on each lane (in parallel) to recover the transmitted bits. These 4 pairs of ADC and MLSE can be implemented on a single application specific integrated circuit (ASIC).

\subsection{Simulation results and discussion}

Fig. 4 presents the required optical power (at the Rx input) as a function of SSMF length (with $D=20\left[\frac{p s}{n m \cdot k m}\right]$ ).

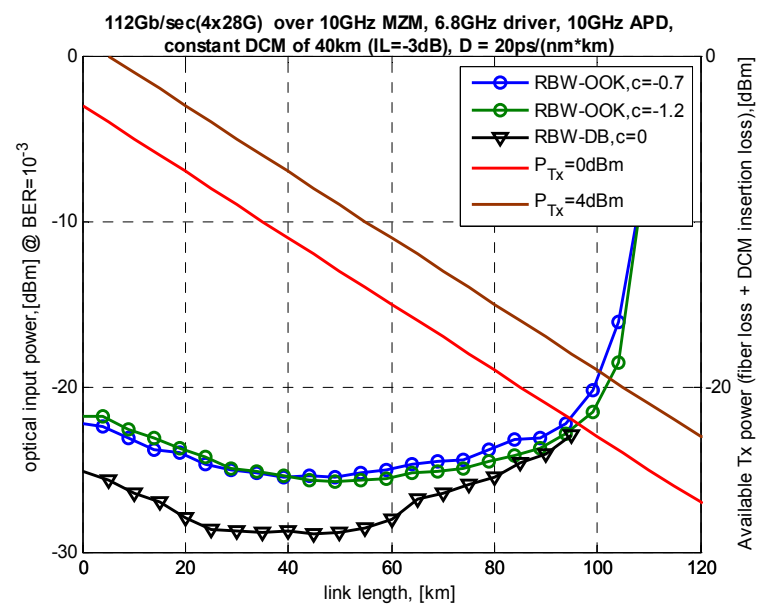

Fig. 4. Required input power and available power vs. link length for RBW-OOK and RBWDB transmission

The red and brown straight lines show the available optical input power at the APD input for $0 \mathrm{dBm}$ and $4 \mathrm{dBm}$ transmission respectively. A constant DCM, compensating for $40 \mathrm{~km}$ fiber with insertion loss (IL) of $3 \mathrm{~dB}$ is used. It is clear that chirped RBW-OOK (with 10G components at the both sides) allows $80-90 \mathrm{~km}$ transmission (without amplification), provided that $40 \mathrm{~km}$ DCM and FEC are used. Moreover, the available power is between 5$9 \mathrm{~dB}$ higher than the required one. The main reason for this improved sensitivity is using the APD instead of PIN photodiode. The MLSE is capable to compensate for ISI introduced by the reduced bandwidth components and the residual portion of $\mathrm{CD}$ that is not compensated optically by DCM.

On contrary to the OOK formats, chirping the duobinary signal will not give any benefit for combating CD. Furthermore, this chirping, in fact, serving as a phase distortion, may even harm the DB signal (Røyset \& Hjelme, 1998). In fact, since the transmitted information is also encoded in the phase of the transmitted optical field, chirping crucially destructs the signal structure. Therefore, in the case of DB transmission $c=0$ is being used. The black curve on 
Fig. 4 shows that non-chirped RBW-DB modulation format has a superior performance over both chirped RBW-OOK cases.

As a matter of the fact, the ordinate values presented on Fig. 4, cannot be considered as the absolute values, but rather the representative ones. It is a well known fact, that the required optical power that ensures some nominal BER, in optically non-amplified system is directly related to the sensitivity of the photo-detector being used. Hence, to achieve more accurate results in a specific system, concrete APD parameters should be considered. The above scenario was obtained with APD, having a sensitivity of $-30 \mathrm{dBm}$ (for $B E R=10^{-9}$ ), which is typical for 10G APDs.

\subsection{Section summary}

In this section, a simple and low cost solution for "extended short reach" $112 \mathrm{~Gb} / \mathrm{sec}$ transmission was proposed. To achieve $112 \mathrm{~Gb} / \mathrm{sec} 4$ different wavelength slots are occupied, whereas the data rate in each slot is $28 \mathrm{~Gb} / \mathrm{sec}$ (including $12 \%$ FEC overhead). Two novel modulation formats: RBW-OOK and RBW-DB are examined, where low cost 10G components (instead of 28G) are used, and MLSE detection technique is applied to recover the data from each sub-channel (slot). The MLSE compensation is carried out in the digital domain, when the 4 signals are digitized using four 5-bit ADCs, operating at 28Gsamples/sec (sampling at the baud rate). Due to its higher sensitivity, 10G APD O/E front end is used, and a constant DCM compensating for $40 \mathrm{~km}$ of SSMF was applied. The ADC and MLSE functionality can be effectively implemented on a single ASIC.

Due to such a low transmission distances, major goal of a system design is cost and complexity reduction, at the expense of sub-optimal, but still applicable, performance. Although RBW-DB shows slightly better $(2-4 \mathrm{~dB})$ performance than chirped RBW-OOK, it requires a more complex and highly accurate transmitter in order to construct the correct $\mathrm{DB}$ signal, and a more expensive Mach-Zehnder modulator to allow modulation of the optical phase and zero chirp. MLSE can compensate for the lack of accuracy of the optoelectronic components, e.g., mismatches of cutoff/order of the DB driver and filter, in addition to the CD compensation.

Since this solution is based on 10G components it can be directly implied on the already existing systems, by replacing the simple HD slicer, by the aforementioned ASIC. Due to the use of the 10G APDs, this is the only kind of 4-wavelength $112 \mathrm{~Gb} / \mathrm{sec}$ transmission system that allows extended reach of up to $80 \mathrm{~km}$ of an SSMF, based on low cost $10 \mathrm{G}$ components.

\section{Metro transmission}

In metro and regional fiber links, the length of the optical channel typically reaches no more than $1000 \mathrm{~km}$. Even with fiber's relatively small loss $\left(\alpha=0.2\left[\frac{d B}{\mathrm{~km}}\right]\right.$ for a SSMF), the signal cannot propagate through it without optical amplification. Moreover, at such high rates the uncompensated transmission can be done only for a few kilometres, hence CD compensation is required. CD compensation can be done in the optical domain or in the electronic domain or a combination of both. In the sequel, two metro- transmission systems 
are proposed. The first can be viewed as an upgrade to the existing direct detection networks, while the latter is based on coherent detection and is suitable for future deployment.

\subsection{Multi-wavelength $112 \mathrm{~Gb} / \mathrm{sec}$ transmission with direct detection and MLSE}

\subsubsection{System description}

In most currently deployed systems (brown field), the former approach is used: the channel is divided into spans (or segments) of $80 \mathrm{~km}$, whereas every span consists of a portion of a SSMF, followed by a DCF and an EDFA as shown on Fig. 5.

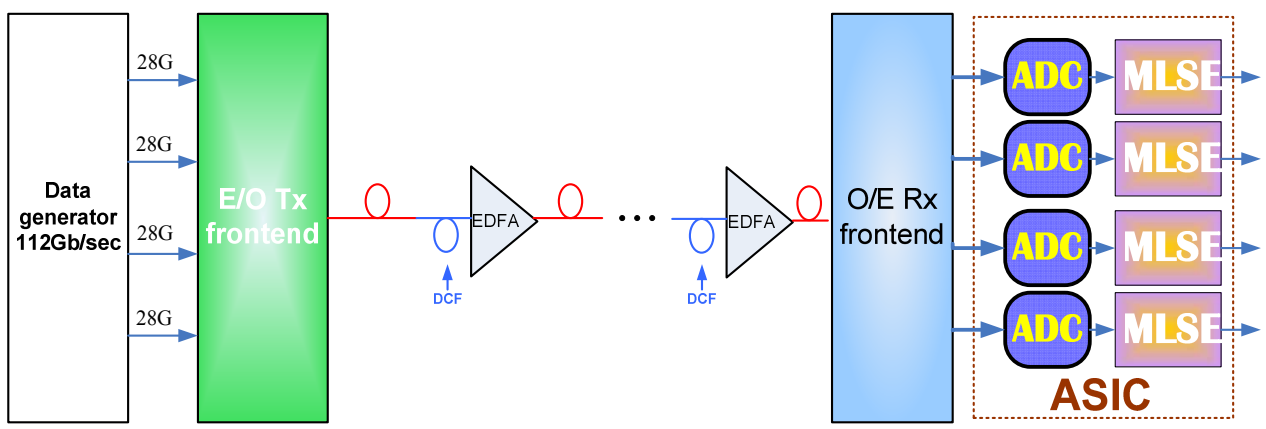

Fig. 5. Multi-wavelength $112 \mathrm{~Gb} / \mathrm{sec}$ direct detection metro transmission system

Pure optical compensation is not financially justified, since different channels have different lengths. DCFs compensate for a fixed amount of dispersion, whereas the residual CD is typically compensated with TDC. It is proposed to employ an electronic MLSE compensation, which adaptively tunes to the uncompensated residual portion of $C D$, rather than using an expensive TDC. The high level block diagram of the proposed 4-wavelength $112 \mathrm{Gbps}$ direct detection metro transmission system is presented on Fig. 5. The O/E and $\mathrm{E} / \mathrm{O}$ frontends are identical to those from previous section, and simple RBW-OOK and RBW-DB modulation formats are examined.

\subsubsection{Simulation results and discussion}

To investigate the tolerance of the presented system to the residual chromatic dispersion and first order PMD, extensive Monte-Carlo simulations were done. 400,000 bits were randomized at each run to ensure sufficient statistics for a pre-FEC BER value of $10^{-3}$.

$\mathrm{CD}$ tolerance versus required optical signal to noise ratio (OSNR) of the two examined modulation formats for different $\mathrm{Tx}$ and Rx filter configurations is presented in Fig. 6 (a). Conventional hard decision (HD) results (dashed lines) were also added for the sake of comparison.

It is clearly observed from Fig. 6 (a) that the combination of reduced bandwidth components at the Tx together with MLSE extends the tolerance to residual dispersion for all examined modulation formats, as compared to a corresponding conventional HD receiver. The main reason for this is the lower analog bandwidth of the transmitted signals. In addition, it is a 
well known fact, that DB modulation has inherent improved CD tolerance due destructive interference between the adjacent symbols, smeared by the CD.

However, at zero CD the required OSNR for the nominal BER in RBW-OOK is $4.5 \mathrm{~dB}$ higher than in DB and RBW-DB. It can be explained by the fact that in DB the combined effect of differential precoding and heavy low-pass filtering at the Tx side is "undone" at the Rx side by the photo detector, effectively eliminating the major portion of (controlled) ISI introduced at the Tx. Basically, the square-law operation at the Rx side doubles the bandwidth of the signal, practically removing the controlled ISI. Thus, on one hand, in RBW-DB, the analog bandwidth of the received signal is reduced by the 10G frontend. On the other hand, the MLSE recovers the bits back, from this filtered signal.

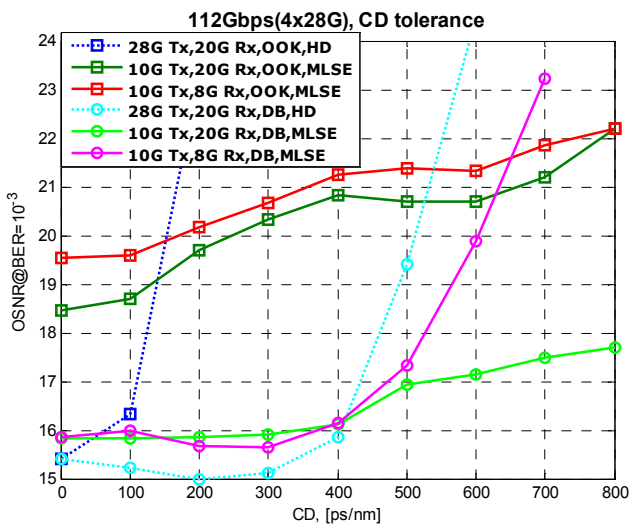

(a)

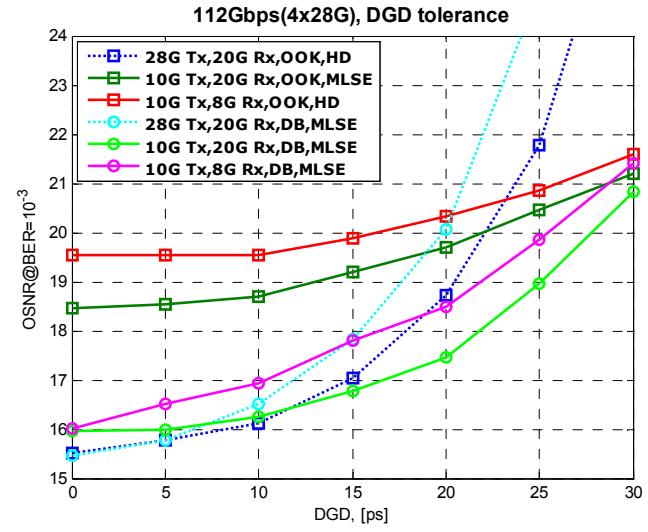

(b)

Fig. 6. (a) CD and (b) DGD tolerance of $112 \mathrm{~Gb} / \mathrm{sec} 4$-wavelength direct detection system

For $C D$ of up to $650\left[\frac{p s}{n m}\right]$, when the overall signal memory length is less than 4 unit intervals (UI), which is also the MLSE memory depth, RBW-DB (magenta circle curve) is more tolerant to chromatic dispersion than RBW-OOK (red square curve). Moreover, CD tolerance can be further improved by extending the bandwidth in the Rx side, as can be seen in the square blue curve. This improvement stems from the fact, that FBW receiver preserves almost the full bandwidth, resulting from Pointing vector calculation during the $\mathrm{O} / \mathrm{E}$ conversion, thus significantly reducing the overall signal memory. However, extending the $\mathrm{Rx}$ bandwidth in the RBW-OOK case provides minor improvement, since ISI introduced at the Tx side is not controlled in any manner at the Rx side, on contrary to its duobinary counterpart. In turn, the overall ISI is weaker in the FBW receiver case than in the RBW receiver case, thus the FBW receiver outperforms the RBW one.

DGD tolerance of the above modulation formats is presented on Fig. 6 (b). Similarly to CD tolerance behavior, it is observed that MLSE detection of all proposed modulation formats, provides better immunity to first order PMD, as compared to hard decision. 
Fig. 6 (b) reveals that RBW-DB modulation formats are more tolerant to first order PMD, than RBW-OOK format. It is worth to emphasize, that since the ISI that results from PMD 'appears' in the signal only during the photo-detection process, both OOK and DB have to 'deal' with the same effect. However, since in duobinary case the controlled ISI is almost completely removed through square-law detection, the resulting waveform is 'cleaner' (from ISI), than its OOK counterpart. Increasing the analog bandwidth at the receiver, earns another $0.5-1 \mathrm{~dB}$ of DGD tolerance in both cases.

\subsubsection{Summary}

In this sub-section, it was shown how to achieve efficient and low cost $112 \mathrm{~Gb} / \mathrm{sec}$ transmission in existing 10G brown field systems. The tradeoffs between occupied bandwidth (4-wavelength slots), system performance and cost are introduced. It is proposed to use an existing, previous generation, low cost 10G opto-electronic components. Performance implications of this intentional bandwidth reduction are examined for the novel RBW-OOK and RBW-DB modulation formats. Parallel MLSE detection of the four lanes, sampled at 28Gsamples/sec is used, to compensate for both channel impairments (CD and PMD) and the reduced bandwidth optical components. It should be mentioned that in light of the presented performance, additional constant DCM module (corresponding roughly to $40 \mathrm{~km}$ compensation) may still be needed, depending on the amount of residual CD in the link. In fact, a combination of MLSE and constant DCM may replace expensive TDC in current brown field networks.

\section{2 "Coherent Metro": Single carrier $112 \mathrm{~Gb} / \mathrm{sec}$ transmission with coherent detection and MLSE}

Sub-section 5.1 described a cost effective solution for upgrading existing (brown field) metro systems for 100G transmission, based on MLSE and low cost optical components. However, if a new fiber systems, (green field), an alternative detection scheme should be considered. The latter is called coherent detection, and in fact it is a more complex and expensive scheme. On the other hand, it introduces significant advantages. For example, since, on contrary to direct detection, in coherent detection the full information about the phase and the amplitude of the optical field is recovered, entire digital compensation of transmission impairments (e.g., CD and PMD) is possible. This, in turn, means that no optical compensation is required, the number of optical amplifiers is reduced, and the available OSNR in the link is increased. Moreover, higher order modulation formats can be used (without significant increase in cost of $\mathrm{E} / \mathrm{O}$ and $\mathrm{O} / \mathrm{E}$ components), resulting in higher spectral efficiency and better performance as compared to direct detection system.

Working at ultra high bit rates of $100 \mathrm{~Gb} / \mathrm{sec}$ and beyond, gives rise to new bottlenecks in the electronic domain: high speed $A D C$ s and massive parallel digital signal processing (DSP) engines are required. To the best of the authors knowledge, the fastest ADC available today (2011) are of 65Gsamples/sec. According to Nyquist criterion, it is able to sample and fully recover signals with $32.5 \mathrm{GHz}$ analog bandwidth. Therefore, aiming to a single carrier transmission, the required bandwidth of the received signal should also be $32.5 \mathrm{GHz}$. To fulfill these requirements for $100 \mathrm{~Gb} / \mathrm{sec}$ transmission, advanced modulation formats with richer vocabulary must be adopted. 
It is a well known relationship between the vocabulary size and the analog bandwidth of the transmitted signal. The higher the vocabulary size $M$, for the given bit rate $R_{b}$, the lower is the symbol rate $R_{s}$ (Proakis, 1995):

$$
R_{s}=\frac{R_{b}}{\log _{2} M}
$$

Hence the bandwidth of the transmitted signal, which is proportional to the symbol rate, also (inversely) scales with the logarithm of $M$. On the other hand, when comparing between two modulation formats, one with small $M=M_{1}$ and the other with larger vocabulary size $M_{2}>M_{1}$, given the same average transmitted power, the former will definitely perform better. The reason for this is that during the decision process, it is harder to distinguish between $M_{2}$ constellation points, spread over the constant region, than doing the same task for $M_{1}$ points. Thus, for a given bit rate, the optimal vocabulary size is determined by the lowest $M$, that provides the required analog bandwidth, as dictated by the maximal ADC sampling rate and the shaping pulse.

Another advantage of coherent detection is doubling the data rate per every channel slot. This is achieved by using the two orthogonal polarization axes to transmit two independent complex signals occupying the same frequency band simultaneously. Therefore, the optimal modulation format, in the sense described in the previous paragraphs, for $100 \mathrm{~Gb} / \mathrm{sec}$ transmission is dual-polarization quadrature phase shift keying (DP-QPSK) with $M=16$. The conclusion of this theoretical discussion was experimentally proven for coherent fiber optical system by (Roberts et al., 2009) for a lower bit rate of $46 \mathrm{~Gb} / \mathrm{sec}$.

Bearing in mind that the raw bit rate is FEC dependent, using the above 65Gsamples/sec ADC, one can transmit 4 independent lanes of net $25 \mathrm{~Gb} / \mathrm{sec}$ yielding $100 \mathrm{Gbps}$ with $30 \%$ FEC overhead assuming the oversampling ratio of 2 . However, focusing on metro transmission ranges (up to $1000 \mathrm{~km}$ ), where the available OSNR in the link is higher than in long-haul links, significant relaxation of ADC sampling rate and DSP operating speed requirements can be achieved, compromising slightly in system performance. Firstly, the powerful, complex and power hungry $30 \%$ overhead soft-decision FEC (SD-FEC) can be replaced by the slightly weaker (Optical Interworking Forum [OIF], 2010) but simple 12\% overhead hard-decision FEC (HD-FEC) with significantly smaller power dissipation. Secondly, since sampling the received signal at symbol rate (i.e. taking only one sample per each symbol period) forms sufficient information (or statistic) for successful recovery of transmitted data (Proakis, 1995), significant relaxation on the ADC operation speed can be achieved. In order not to violate Nyquist criterion, an anti-aliasing filter (AAF) must be used prior the ADC. This AAF introduces additional ISI, which cannot be optimally equalized by means of finite impulse response (FIR) filters. It is shown by (Gorshtein, et al., 2010) that using an MLSE compensates for the AAF effect, enabling the reduction by a factor of two of the ADC sampling rate and DSP processing speed, all together leading to significantly lower complexity and power saving.

\subsubsection{System model}

High level block diagram of the $112 \mathrm{~Gb}$ / sec coherent DP-QPSK optical fiber communication system is presented on Fig. 7. 


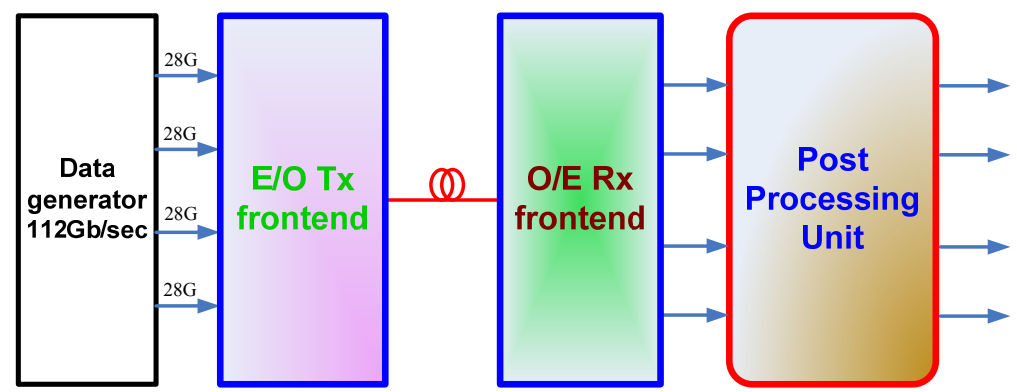

Fig. 7. 112Gb/sec DP-QPSK coherent detection metro transmission system

E/O Tx and O/E Rx frontends are depicted on Fig. 8 (a) and (b) respectively.

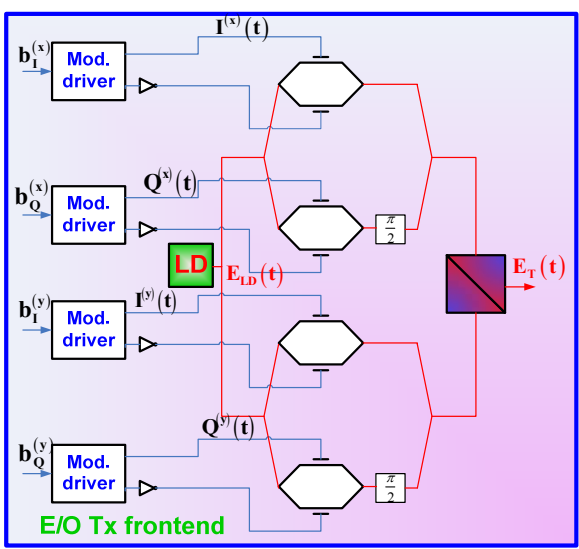

(a)

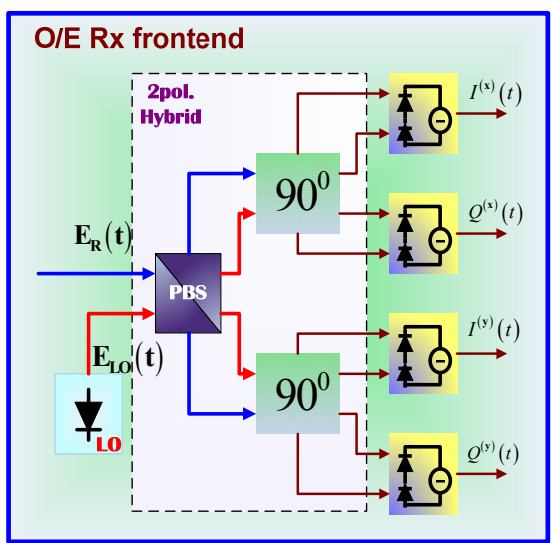

(b)

Fig. 8. 112Gb/sec coherent DP-QPSK (a) E/O Tx frontend and (b) O/E Rx frontend

Four independent 28G data streams are driving four MZM, operating in a push pull configuration. In two out of four MZM outputs additional $\frac{\pi}{2}$ phase shift is generated, resulting in two QPSK signals, which are combined by a polarization beam splitter (PBS) to construct a transmitted 112Gb/sec DP-QPSK signal, as can be seen from Fig. 8 (a). At the Rx side Fig. 8 (b), the received signal is mixed with the signal coming from local oscillator (LO). The mixing is carried out with help of 2-polarisation optical hybrid (in turn consisting of a pair of 90 degrees hybrids and a PBS), followed by a four balanced detectors. The output photocurrents correspond to the in-phase and quadrature components at both polarizations, conveying the full information about the amplitude and the phase of the received optical field.

\subsubsection{Brief description of post-processing unit algorithms}

The post processing unit (PPU), for compensation of linear transmission impairments in coherent $112 \mathrm{~Gb} /$ sec DP-QPSK system, is shown on Fig. 9. 


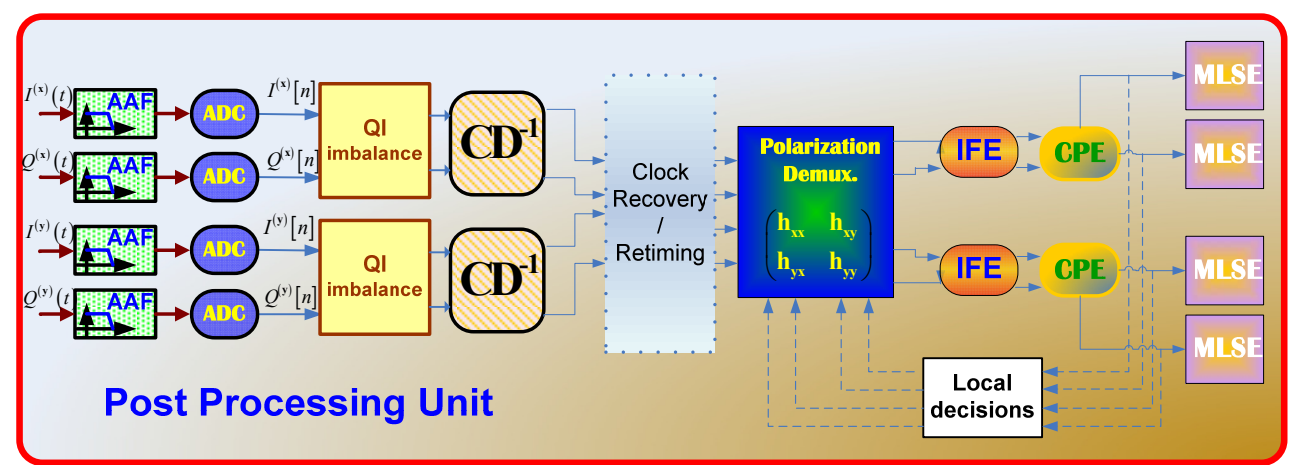

Fig. 9. PPU for $112 \mathrm{~Gb} / \mathrm{sec}$ DP-QPSK coherent detection metro transmission system

At the input of the PPU there are four lanes corresponding to the in-phase and quadrature signals, which can be expressed analytically as follows:

$$
\begin{aligned}
& I_{\mathbf{R}}^{(\mathbf{x})}(t)=K \cdot\left(\begin{array}{l}
\cos ^{2} \psi\left[\sum_{l} r_{l}\left(t-\frac{\tau}{2}\right) e^{j \theta_{l}^{(\mathbf{x})}}\right]+\sin ^{2} \psi\left[\sum_{l} r_{l}\left(t+\frac{\tau}{2}\right) e^{j \theta_{l}^{(\mathbf{x})}}\right]- \\
-\frac{1}{2} \sin 2 \psi\left[\sum_{l} r_{l}\left(t-\frac{\tau}{2}\right) e^{j \theta_{l}^{(\mathbf{y})}}-\sum_{l} r_{l}\left(t+\frac{\tau}{2}\right) e^{\left.j \theta_{l}^{(\mathbf{y})}\right]}+I_{\mathbf{N}}^{(\mathbf{x})}(t)\right.
\end{array}\right)+\left(\begin{array}{l}
\cos ^{2} \psi\left[\sum_{l} r_{l}\left(t+\frac{\tau}{2}\right) e^{j \theta_{l}^{(\mathbf{y})}}\right]+\sin ^{2} \psi\left[\sum_{l} r_{l}\left(t-\frac{\tau}{2}\right) e^{j \theta_{l}^{(\mathbf{y})}}\right] \\
-\frac{1}{2} \sin 2 \psi\left[\sum_{n} r_{l}\left(t-\frac{\tau}{2}\right) e^{j \theta_{l}^{(\mathbf{x})}}-\sum_{l} r_{l}\left(t+\frac{\tau}{2}\right) e^{j \theta_{l}^{(\mathbf{x})}}\right]
\end{array}\right)+I_{\mathbf{N}}^{(\mathbf{y})}(t)
\end{aligned}
$$

where $I_{\mathbf{R}}^{(\mathbf{x})}(t)$ and $I_{\mathbf{R}}^{(\mathbf{y})}(t)$ represent complex baseband currents in each polarization. Equation (5.2) accounts for a linear channel, including the following effects: $C D$, first order $\mathrm{PMD}$, laser phase noise at both $\mathrm{Tx}$ and $\mathrm{Rx}$ (local oscillator) lasers, intradyne reception and additive Gaussian noise formed by optical amplifiers. In Equation (5.2) $e^{j \theta_{l}^{(\mathbf{x})}}, e^{j \theta_{l}^{(\mathbf{y})}}$ represent the $\mathbf{x}$ - and y-polarization information symbols, and $I_{\mathbf{N}}^{(\mathbf{x})}(t), I_{\mathbf{N}}^{(\mathbf{y})}(t)$ denote the complex noise terms respectively. The effect of PMD in (5.2) is expressed through $\psi$ (the angle between the input state of polarization in the fiber and the principal state of polarization) and $\tau$ being the DGD between the polarization modes. The influences of CD, phase noises and intradyne frequency are represented by the shaping pulse:

$$
r_{l}(t)=\left(h_{C D} *\left[g(t-l T) e^{j \phi_{\mathrm{T}}(t)}\right]\right) \cdot e^{j\left[\omega_{I F} t+\phi_{\mathbf{R}}(t)\right]}
$$


where $\phi_{\mathrm{T}}(t)$ and $\phi_{\mathbf{R}}(t)$ represent the phase noises at the Tx and Rx respectively, $h_{C D}(t)$ being the impulse responses accounting for $\mathrm{CD}, \omega_{I F}$ denotes the angular intradyne frequency, and $g(t)$ is the shaping pulse at the transmitter which is assumed non-return to zero (NRZ) throughout this manuscript. In Fig. 9, each lane, corresponding to the real and imaginary parts of $\mathrm{x}$ - and $\mathrm{y}$ - polarization in (5.2), is filtered out by an AAF which is modeled as a $5^{\text {th }}$ order Butterworth LPF. In turn, the signal at each lane is sampled and quantized by an ADC at sampling rate of 28 Gsamples/sec with 5-bit resolution.

The proposed PPU is similar to those presented in (Ip \& Kahn, 2007; Fludger et al. 2008; Kuschnerov et al., 2009). The important differences are: (a) the incoming signal bandwidth is intentionally reduced by the AAFs to comply with the Nyquist sampling theorem, (b) sampling and DSP are being done at symbol rate, and (c) the AAF-related introduced ISI is compensated by post processing MLSE.

The lack of orthogonality between I- and Q-signals, stemming mainly from non-perfect 2pol. hybrid, is compensated by the I-Q imbalance blocks (Savory, 2010). CD compensation is performed by $\mathrm{CD}^{-1}$ blocks by sampling (1.3) and using Fast Fourier Transform together with overlap-add/save method. After removing a bulk amount of chromatic dispersion, clock recovery unit corrects the mismatch between the Tx and Rx clocks, and interpolating the input signal to the optimal sampling point. Polarization Demux block in Fig. 9 carries out both polarization demultiplexing and PMD compensation functionalities. IFE (Leven, 2007) and CPE (Viterbi A.J. \& Viterbi A.M., 1983) blocks perform intradyne frequency estimation and carrier phase estimation to compensate for frequency mismatch and lasers' phase noises respectively.

\subsubsection{Simulation results and discussion}

Combined CD and PMD tolerance of $112 \mathrm{~Gb} / \mathrm{sec}$ coherent detection DP-QPSK system is presented on Fig. 10.

Here, polarization and $\mathrm{CD}$ effects have been examined, while the same laser serves as the local oscillator and the transmit laser, i.e. no frequency mismatch occurs. Carrier phase estimation and timing recovery were assumed ideal. In fact, the effect of sampling phase is significantly reduced due to the AAF-MLSE combination as explained in (Gorshtein, 2010). Yet, an optimization is required due to the least mean squares (LMS) presence.

Each Monte-Carlo simulation set includes 400,000 random bits. The CD equalizer obeys a zero forcing criterion, and is implemented in the frequency domain, while the CD value is predetermined. The number of states in the MLSE is 16. Histogram estimation method is used for channel estimation where a novel blind equalization scheme is used, as described in section 2 (and in (Gorshtein, 2010)).

Fig. 10 reveals that the proposed system successfully compensates for $20,000\left[\frac{\mathrm{ps}}{\mathrm{nm} \cdot \mathrm{km}}\right]$ of CD (corresponding to $1000-1200 \mathrm{~km}$ fiber) together with up to $100 \mathrm{ps}$ of DGD. The overall penalty of about $2 \mathrm{~dB}$ as compared to its back-to-back (b2b) scenario, for worst case scenario is observed. The main reason for this penalty comes from the AAF (Gorshtein, 2010) whereas MLSE engine is used in each lane to struggle this intentionally introduced ISI in the most optimal manner. 


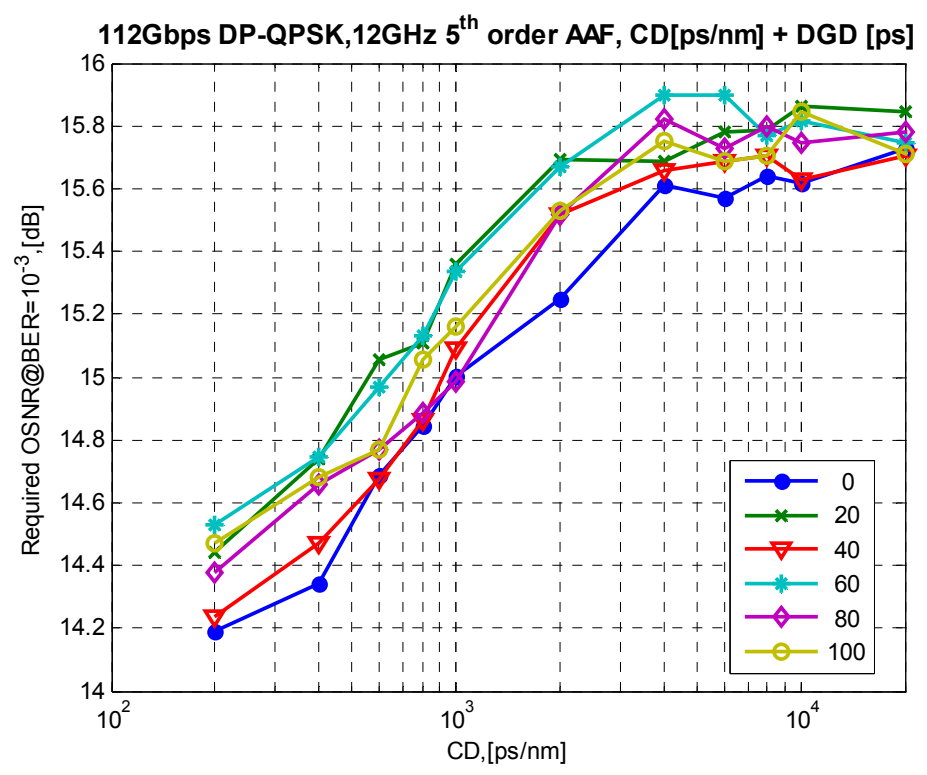

Fig. 10. Combined CD and PMD tolerance of $112 \mathrm{~Gb} / \mathrm{sec}$ DP-QPSK coherent detection metro transmission system

\subsubsection{Summary}

Sub-section 5.2 presented a "Coherent Metro" MLSE-based solution for $112 \mathrm{~Gb} / \mathrm{sec}$ transmission, which can be used in new deployed systems (green field). The considerations of choosing optimal modulation format were discussed. DP-QPSK modulation format is the most suitable for metro transmission in the proposed system. The DSP that is required in coherent systems, briefly described above, is significantly more complicated than as compared to a direct detection system. Major bottlenecks of a coherent system are requirements for tremendous $\mathrm{ADC}$ sampling rate, operation speed of the aforementioned DSP and associated complexity and power dissipation. Significant relaxations of all above can be achieved by introducing AAF, allowing efficient practical implementation, whereas accompanying ISI can be compensated by the MLSE with only $2 \mathrm{~dB}$ penalty (as compared to b2b) for 100ps DGD in 1200km link.

\subsection{Section summary and conclusions}

In this section ultra-high speed $112 \mathrm{~Gb} / \mathrm{sec}$ systems for brown and green field applications were proposed. In the brown field, 4-wavelength direct detection is suggested, where reduced bandwidth, already existing and mature, 10G components are used for $28 \mathrm{~Gb} / \mathrm{sec}$ transmission in each lane. Since most deployed 10G systems use simplest modules at both Tx and Rx sides, new RBW-OOK and RBW-DB modulation formats, which can be transmitted and received in such a network, were proposed and examined. Four MLSE engines are used to compensate for ISI, introduced by the reduced bandwidth components, and for performance improvement. MLSE reception of the proposed 
modulation formats extends the link tolerance to residual CD and first order PMD, as compared to conventional HD receiver. In many brown field 10G metro networks, the available OSNR is in the range 18-24dB. RBW-DB requires lower OSNR to achieve the same BER performance as compared to RBW-OOK, due to the controlled ISI that is inherent in DB format. Using full bandwidth receiver with RBW-DB transmission can dramatically reduce the OSNR requirement. Yet, for all examined modulation formats, the extended CD tolerance is limited to less than $70 \mathrm{~km}$. For full compensation of $80 \mathrm{~km}$ spans an additional fixed optical compensation module is needed.The proposed solution significantly reduces system cost both due to the use of previous generation 10G components and by replacing expensive tunable dispersion compensation element by a constant fixed dispersion compensation module. This approach transfers the tuneability task to the digital domain, executed by the MLSE engine.

When designing a new green field metro network, coherent detection is preferred. The received photocurrents are proportional to the transmitted optical field components (amplitude and phase) enabling full digital compensation of transmission impairments, e.g., CD, PMD, non-perfect and non-synchronized lasers and so on. DP-QPSK modulation format is examined, due to its higher spectral efficiency as compared to binary formats on one hand, and better performance than modulation formats with richer vocabulary on the other hand. Major bottlenecks of this approach are ultra-high sampling rate, DSP operating speed, complexity and power dissipation. Most coherent 100G systems investigated today employ two-fold oversampling, requiring ultra-fast ADCs. Noting that in metro systems, OSNR of 16-18 dB can be obtained, ADC sampling rate, DSP complexity and power can be dramatically reduced, by employing the baud rate sampling, preceded by the appropriate AAF. MLSE is used to compensate for ISI, which is intentionally introduced by heavy antialiasing low pass filtering. It is shown that the proposed system can tolerate CD of about $1200 \mathrm{~km}$ together with 100ps DGD, when only minor $(2 \mathrm{~dB})$ penalty is present due to the introduction of the AAF.

\section{Long-haul transmission}

The link lengths of long-haul transmission systems is $1200 \mathrm{~km}$ and above. Due to such high distances and attenuation, many optical amplifiers are required. Therefore the available OSNR in the link becomes more deficient. Hence, to achieve the required performance, the system should perform very close to the theoretical optimum. In turn, effective $112 \mathrm{~Gb} / \mathrm{sec}$ transmission with coherent detection would be the most promising solution. Furthermore, since nearly optimal performance is required, all the DSP equalization techniques, sketched in the previous section, must operate on (two-fold) oversampled signals, requiring higher speed ADC (of the order of 64Gsamples/sec for DP-QPSK). In this case, the MLSE is not needed, as such oversampled systems can recover the full performance as indicated both theoretically (IP \& Kahn, 2007) and shown experimentally by various groups: (Fludger et al., 2008; Kushnerov et al., 2009; Savory 2010) and references therein. Since in oversampled systems, AAF practically does not introduce such a severe ISI (as compared to the baud rate sampling scenario presented in the previous section), MSLE is not obligate, and even obsolete due to its complexity and power consumption (especially at such a high operating rate). 


\section{Conclusion}

High speed (100G b/sec and beyond) optical communication undergoes a revolution nowadays. Together with increasing the volume of transmitted information per second, currently deployed systems become severely limited by CD and PMD. New cost effective, low power and low complexity solutions are desired. Depending on the transmission range, different system design and equalization schemes are required. In general, digital compensation of transmission impairments is more cost effective as compared to optical compensation, at the expense of power dissipated by the associated DSP ASIC. High speed ADCs and equalization post processing form main bottlenecks for this kind of solutions.

In this chapter novel MLSE-based approaches for $112 \mathrm{~Gb} / \mathrm{sec}$ transmission were proposed for "extended short reach" and metro links. The optical fiber was described from the communication theory system point of view. Various trade-offs between spectral efficiency, complexity, power and cost for each class of the links were presented and discussed in details. The principles of MLSE equalization were outlined and a new blind channel estimation technique was introduced. In addition, a method for upgrading existing 10G links to achieve $112 \mathrm{~Gb}$ /sec using 4-wavelengths, based on "previous generation" low cost $10 \mathrm{G}$ components instead of $28 \mathrm{G}$ components was proposed. Two novel modulation formats RBW-OOK and RBW-DB were examined for different short and metro links categories, based on direct detection and MLSE equalization. Coherent detection scheme for green field single carrier $112 \mathrm{~Gb} / \mathrm{sec}$ DP-QPSK metro applications with reduced power consumption and lower complexity was proposed.

For "extended short reach" links coherent detection is not justified. Hence 4-wavelngth $112 \mathrm{~Gb} / \mathrm{sec}$ transmission based on direct detection and simplest modulation formats is preferred. The proposed solution benefits from the fact that 10G APDs are available, whereas their 28G counterparts are still not available. Therefore, the proposed method, based on MLSE detection of RBW-OOK and RBW-DB modulation formats, is a very attractive solution possible with appropriate combination of cost, complexity and power for $112 \mathrm{~Gb} / \mathrm{sec}$ transmission in these ranges.

$10 \mathrm{~Gb} / \mathrm{sec}$ brown field transmission with optical dispersion compensation and direct detection is very popular in metro links. To achieve $112 \mathrm{~Gb} / \mathrm{sec}$, currently deployed $10 \mathrm{G}$ systems may be used. 4 -wavelength slots can be dedicated to carry $112 \mathrm{~Gb} / \mathrm{sec}$, and existing low cost $10 \mathrm{G}$ components can be used for $28 \mathrm{~Gb} / \mathrm{sec}$ transmission in each slot. Depending on the link length, available OSNR and the sensitivity of the photo-detectors several variants of RBW-OOK and RBW-DB can be used.

Extending the reach for $112 \mathrm{~Gb} / \mathrm{sec}$ metro transmission, and later on in green field networks, cost effective solutions can be achieved by employing "Coherent Metro" systems. Coherent detection allows the entire removal of DCFs, effectively increasing the available OSNR in the link. The task of channel equalization is performed in the digital domain, provided that full information of the amplitude and phase of the optical field is recovered during the detection process. The combination of AAF and MLSE is proposed to form power efficient and cost effective solution for single carrier $112 \mathrm{~Gb} / \mathrm{sec}$ DP-QPSK transmission. This method provides significant relaxation on ADC sampling rate and operation speed of the following equalization of transmission impairments, at the expense of additional $2 \mathrm{~dB}$ of OSNR penalty which seems acceptable in metro links. 
Since in long-haul transmission every single $\mathrm{dB}$ of OSNR is critical, there is no room for OSNR - ADC speed compromise, and the incoming signal must be oversampled. In this case the MLSE is obsolete, due to extra ASIC complexity and power dissipation.

\section{References}

Agazzi, O. et al. (2005), Maximum-Likelihood Sequence Estimation in Dispersive Optical Channels, Journal of Lightwave Technology, Vol. 23, No.2, (February 2005), pp.749 763

Agrawal, G. (2002). Fiber optic communications systems, John Wiley \& Sons, Inc., ISBN 0-47121571-6, USA

Fludger, C. et al. (2008), Coherent Equalization and POLMUX-RZ-DQPSK for Robust 100GE Transmission, Journal of Lightwave Technology., Vol. 26, No. 1, (January 2008), pp. 131-141.

Foggi, T. et al. (2006), Maximum-Likelihood Sequence Detection With Closed-Form Metrics in OOK Optical Systems Impaired by GVD and PMD, Journal of Lightwave Technology, Vol. 24, No.8, (August 2006), pp.3073 - 3086

Gorshtein, A. et al. (2010), Coherent Compensation for 100G DP-QPSK with One Sample per Symbol Based on Anti-Aliasing Filtering and Blind Equalization MLSE, Photonic Technology Letters., Vol.22, No.16, (August 2010), pp. 1208-1210

Ip, E. \& Kahn, J. (2007). Digital Equalization of Chromatic Dispersion and Polarization Mode Dispersion. Journal of Lightwave Technology., Vol.25, No.8, (August 2007), pp. 20332043

Kaminov, I. \& Li, T. (2001). Optical Fiber Telecommunications, Academic Press Elsevier Science, ISBN 0-12-395173-9, San Diego, California, USA

Kuschnerov, M. et al. (2009), DSP for Coherent Single-Carrier Receivers, Journal of Lightwave Technology., Vol. 27, No. 16, (August 2009), pp. 3614-3622.

Leven, A. et al. (2007), Frequency Estimation in Intradyne Reception, Photonic Technology Letters, Vol. 19, No. 6, (March 2007) pp.366-368.

Optical Interworking Forum (May, 2010), 100G Forward Error Correction White Paper, In: OIF-FEC-100G-01.0, 14.05.2011, Available from http://www.oiforum.com/public/documents/OIF_FEC_100G-01.0.pdf

Proakis, J. (1995). Digital Communications, McGraw-Hill, ISBN 0-07-051726-6, Singapore

Roberts, K. et al. (2009), Performance of Dual-Polarization QPSK for Optical Transport Systems, Journal of Lightwave Technology., Vol.27, No.16, (August 2009), pp. 35463559

Røyset, A. \& Hjelme, D. (1998), Symmetry Requirements for 10-Gb/s Optical Duobinary Transmitters, Photonic Technology Letters, Vol.10, No.2, (February 1998), pp. 273275

Savory, S. (2010), Digital Coherent Optical Receivers: Algorithms and Subsystems, IEEE Journal of Selected Topics in Quantum Electronics, Vol. 16, No. 5, (October 2010), pp. 1164-1179

Viterbi, A.J. \& Viterbi, A.M. (1983), Nonlinear Estimation of PSK-modulated Carrier Phase with Application to Burst Digital Transmission, IEEE Transactions on Information Theory, Vol. IT-29, No. 4, (July 1983) pp. 543-551. 
Winzer, P. \& Essiambre, R. (2006). Advanced Optical Modulation Formats, Proceedings of the IEEE, Vol. 94, No. 5, (May 2006), pp. 952-985 


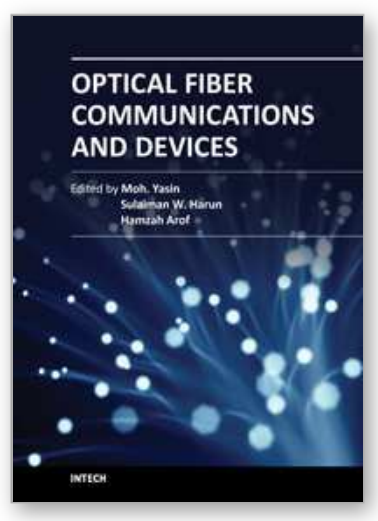

\author{
Optical Fiber Communications and Devices \\ Edited by Dr Moh. Yasin
}

ISBN 978-953-307-954-7

Hard cover, 380 pages

Publisher InTech

Published online 01, February, 2012

Published in print edition February, 2012

This book is a collection of works dealing with the important technologies and mathematical concepts behind today's optical fiber communications and devices. It features 17 selected topics such as architecture and topologies of optical networks, secure optical communication, PONs, LANs, and WANs and thus provides an overall view of current research trends and technology on these topics. The book compiles worldwide contributions from many prominent universities and research centers, bringing together leading academics and scientists in the field of photonics and optical communications. This compendium is an invaluable reference edited by three scientists with a wide knowledge of the field and the community. Researchers and practitioners working in photonics and optical communications will find this book a valuable resource.

\title{
How to reference
}

In order to correctly reference this scholarly work, feel free to copy and paste the following:

Albert Gorshtein and Dan Sadot (2012). Advanced Modulation Formats and MLSE Based Digital Signal Processing for 100Gbit/sec Communication Through Optical Fibers, Optical Fiber Communications and Devices, Dr Moh. Yasin (Ed.), ISBN: 978-953-307-954-7, InTech, Available from:

http://www.intechopen.com/books/optical-fiber-communications-and-devices/advanced-modulation-formatsand-mlse-based-digital-signal-processing-for-100gbit-sec-communication-t

\section{INTECH}

open science | open minds

\author{
InTech Europe \\ University Campus STeP Ri \\ Slavka Krautzeka 83/A \\ 51000 Rijeka, Croatia \\ Phone: +385 (51) 770447 \\ Fax: +385 (51) 686166 \\ www.intechopen.com
}

\author{
InTech China \\ Unit 405, Office Block, Hotel Equatorial Shanghai \\ No.65, Yan An Road (West), Shanghai, 200040, China \\ 中国上海市延安西路65号上海国际贵都大饭店办公楼 405 单元 \\ Phone: +86-21-62489820 \\ Fax: +86-21-62489821
}


(C) 2012 The Author(s). Licensee IntechOpen. This is an open access article distributed under the terms of the Creative Commons Attribution 3.0 License, which permits unrestricted use, distribution, and reproduction in any medium, provided the original work is properly cited. 